\title{
Nuclear localization of NF-ATc by a calcineurin-dependent, cyclosporin-sensitive intramolecular interaction
}

\author{
Chan R. Beals, Neil A. Clipstone, ${ }^{1}$ Steffan N. Ho, and Gerald R. Crabtree ${ }^{2}$ \\ Howard Hughes Medical Institute, Department of Developmental Biology, Stanford University, \\ Stanford, California 94305-5428 USA
}

The NF-AT family of transcription factors participates in the regulation of early immune response genes such as IL-2, IL-4, CD40 ligand, and Fas ligand in response to $\mathrm{Ca}^{2+} /$ calcineurin signals initiated at the antigen receptor. Calcineurin activation leads to the rapid translocation of NF-AT family members from cytoplasm to nucleus, an event that is blocked by the immunosuppressive drugs cyclosporin A and FK506. We show that translocation requires two redundant nuclear localization sequences and that one sequence is in an intramolceular association with phosphorserines in a conserved motif located at the amino terminus of each NF-AT protein. Mutation of serines in this motif in NF-ATc both disrupts this intramolecular interaction and leads to nuclear localization, suggesting a model of NF-AT nuclear import in which dephosphorylation by calcineurin causes exposure of two nuclear localization sequences.

[Key Words: Calcineurin; cyclosporin/nuclear localization sequence; protein phosphorylation; tacrolimus]

Received January 14, 1997; revised version accepted February 24, 1997.

Signals initiated at the $\mathrm{T}$ lymphocyte antigen receptor result in the orderly expression of multiple genes in peripheral lymphocytes that direct cell proliferation, immunologic function, and ultimately the programmed cell death of accumulated activated cells. This differentiation program, dubbed activation, requires $\sim 2$ weeks for completion and helps to coordinate the actions of many cell types through the activation of genes that encode cell surface homing receptors, cytotoxic molecules, and many secreted molecules necessary for an effective immune response. The final act of $\mathrm{T}$-cell activation is most commonly cell death, an apparent assisted suicide, mediated largely by the Fas receptor and its ligand for the purpose of removing clones of antigen responsive cells when they are no longer needed (Brunner et al. 1995).

The cell-cell interaction between the polymorphic $\mathrm{T}$ cell antigen receptor on lymphocytes and major histocompatibility/antigen complex ( $\mathrm{MHC}$ ) on antigen-presenting cells activates at least three signaling pathways in $\mathrm{T}$ lymphocytes. One pathway requires Ras and the mitogen-activated protein (MAP) kinases, terminating in the activation of serum response factor (SRF) and the c-fos gene (Downward et al. 1990; for review see Treis-

\footnotetext{
${ }^{1}$ Present address: Department of Microbiology and Immunology, Northwestern University Medical School, Chicago, Illinois 60611 USA.

${ }^{2}$ Corresponding author.

E-MAIL hf.grc@forsythe.stanford.edu; FAX (415) 723-1399.
}

man 1995). A second signaling pathway is the Rac/ Cdc42-dependent control of the Jun kinase or stress-activated protein kinases (Minden et al. 1995). This widely distributed signaling pathway may also play an important role in cytoskeletal changes necessary for lymphocyte function (Stowers et al. 1995). A third essential pathway controls the elevation of intracellular calcium $\left(\left[\mathrm{Ca}^{2+}\right]_{\mathrm{i}}\right)$ and regulates the $\mathrm{Ca}^{2+}$-dependent protein phosphatase calcineurin (Klee et al. 1979).

Calcineurin plays a role in events as diverse as axonal guidance in mammals (Chang et al. 1995) and light responses in plants (Luan et al. 1993) but is also a vital regulatory protein in T-cell activation /Clipstone and Crabtree 1992; O'Keefe et al. 1992; Woodrow et al. 1993; Fruman et al. 1995; Zhang et al. 1996) where it controls the function of the NF-AT (nuclear factor of activated $\underline{T}$ cell) family of transcription factors (Shaw et al. 1988; Clipstone and Crabtree 1992). The medically important immunosuppressive drugs cyclosporin (CsA) and tacrolimus (FK506) specifically inhibit the activity of calcineurin (Liu et al. 1991) and selectively block the nuclear localization of NF-ATc (Flanagan et al. 1991). These drugs prevent the transcriptional activation of a variety of essential early immune response genes, each of which contain binding sites for NF-AT (Shaw et al. 1988; Cockerill et al. 1995; Chuvpilo et al. 1993; Rooney et al. 1995; Goldfeld et al. 1993). To date, a null mutation in one NF-AT family member, NF-ATp, has been reported and shows reduced expression of interleukin-4 (IL-4), Fas li- 
gand and CD40 ligand (Hodge et al. 1996). NF-AT family members appear to be controlled primarily at the level of nuclear localization, as the cytoplasmic form of the protein is competent for both DNA binding and transcription (Flanagan et al. 1991). Although a number of transcription factors including AP-1 and NF- $\mathrm{KB}$ may be affected by cyclosporin (Emmel et al. 1989), direction of NF-ATc to the nucleus by overexpression renders T cells resistant to cyclosporin and independent of a calcium stimulus (Timmerman et al. 1996). The latter observations indicate that NF-ATc nuclear localization may be the major early event controlled by calcineurin and calcium in $\mathrm{T}$ lymphocytes.

Nuclear entry of NF-ATc appears to be accompanied by dephosphorylation (Jain et al. 1993; Ruff and Leach 1995; Loh et al. 1996), and expression of an activated form of calcineurin renders NF-AT-dependent transcription independent of a $\mathrm{Ca}^{2+}$ signal and insensitive to cyclosporin (Woodrow et al. 1993), suggesting that NF-ATc family members are substrates for calcineurin and that dephosphorylation may control NF-ATc localization. Possible proline-directed kinase sites are present in a conserved tandemly repeated motif (SPXXSPXXSPrX$\mathrm{sXtD} / \mathrm{ED} / \mathrm{Eswl}$ ) of unknown function termed the serine/ proline (SP) repeat (Ho et al. 1995; Hoey et al. 1995; Masuda et al. 1995). In addition, a second conserved motif, termed the serine-rich region (SRR) (Ho et al. 1995) of unknown function displays a group of possible kinase sites predicted to be surface exposed.

We have focused our attention on the mechanism of nuclear entry of NF-ATc family members because NFAT translocation appears essential for the activation of early immune response genes in lymphocytes and its inhibition appears to be in part responsible for the immunosuppressive actions of cyclosporin A.

\section{Results \\ Calcineurin appears to be rate-limiting for NF-ATC nuclear entry}

Most tissues express one of the NF-AT family members. A variety of cell types, including lymphocytes and fibroblasts, support the $\mathrm{Ca}^{2+}$-dependent nuclear localization of transfected as well as endogenous NF-ATc family members (Shibasaki et al. 1996). To develop an accurate assay for NF-AT translocation, we expressed NF-ATc in COS cells that, unlike lymphocytes, have abundant cytoplasm and hence allow easier assessment of cytoplasmic and nuclear localization. As with NF-ATc3(4) (Shibasaki et al. 1996), we found that the amino terminus of NF-ATc was sufficient for $\mathrm{Ca}^{2+}$-regulated nuclear import that was blocked by FK506 (Fig. 1B). Transfected NF-ATc moved into the nucleus within 5-15 min after ionomycin treatment (Fig. 1A,B) and moved back out into the cytoplasm within $30 \mathrm{~min}$ of FK506 addition (Fig. 1D). These translocations occurred even if protein synthesis was inhibited (data not shown). The full-length protein behaved similar to a fusion of the amino-terminal 418 amino acid with green fluorescent protein (GFP) (Fig.
lC). This time course of nuclear localization is consistent with that observed with murine lymphocytes activated by antigen presentation (Timmerman et al. 1996) and indicates that COS cells can support physiologic translocation of NF-ATc. As with NF-ATc3(4) (Shibasaki et al. 1996) overexpression of calcineurin enhanced the movement of NF-AT into the nucleus of COS cells (Fig. 1A), suggesting that calcineurin is rate limiting for the movement of both NF-ATc and NF-ATc3 into the nucleus.

\section{Addition of heterologous nuclear localization sequences to NF-ATc results in $\mathrm{Ca}^{2+}$-independent, FK506-resistant nuclear import}

The observation that overexpressed NF-ATc is cytoplasmic in Jurkat $\mathrm{T}$ lymphocytes (not shown) and COS cells suggests that there is not an easily saturated cytoplasmic anchoring protein necessary to retain NF-ATc in the cytoplasm. Transfection of the NF-ATc expression construct over a 200 -fold range of DNA concentration did not result in higher levels of constitutive nuclear localization (data not shown). If NF-ATc was localized by a cytoplasmic anchoring partner, the addition of a fully active nuclear localization sequence (NLS) to NF-ATc should not overcome the cytoplasmic retention of NFATc. Expression of NF-ATc with zero, one, or two copies of the SV40 large T antigen NLS in COS cells results in a progressive increase in constitutive nuclear localization (Fig. 2) which was insensitive to FK506 (data not shown). In contrast, addition of the mutant NLS sequence, NLS-T (Kalderon et al. 1984), to NF-ATc resulted in substantially less nuclear entry. The low level of nuclear localization resulting from inclusion of NLS-T may be attributable to slight activity of this mutant, which, like the wild-type sequence, is enhanced when present in multiple copies (Roberts et al. 1987). These results argue against a mechanism of cytoplasmic localization dependent on a dominantly acting cytoplasmic binding protein.

\section{Two NLSs are necessary and sufficient for NF-ATc nuclear translocation}

We noted four groups of clustered basic residues conserved among NF-ATc proteins that could possibly be NLSs. We tested these sequences for NLS activity by linking them individually to the cytoplasmic exchange factor SOS. Two of these chimeric proteins, SOS-682, incorporating residues 682-685 of NFATc, and SOS-265, incorporating residues 265-267, are localized in the nucleus (Fig. 3). These two conserved regions within NFATc are thus NLSs.

To determine whether these NLSs are required for nuclear import of NF-ATc, each sequence was mutated separately and in combination within the context of fulllength NF-ATc. Mutation of the sequence at 265-267 from KRK to QIL reduced the extent of nuclear localization of NF-ATc in response to ionomycin, but up to $60 \%$ of cells show some $\mathrm{Ca}^{2+}$-dependent nuclear accumula- 
Figure 1. The amino terminus of NF-ATc is sufficient for rapid and calcineurin-dependent cytoplasmic to nuclear translocation. (A) Diagram of the NF-ATc1 protein. NF-ATc family members have a RSD that binds DNA and a conserved amino terminus that contains serine- and proline-containing elements, a SRR, and three repeats (SP1, SP2, SP3). The amino acid boundaries of the SRR and $\mathrm{SP}$ repeats are indicated for human NF-ATcl. The relative positions of two NLSs are also marked. The expressed proteins contain a FLAG epitope tag at the amino terminus. (B) Time course of nuclear translocation of NF-ATc in COS cells. Cells were transiently transfected with NF-ATc (SH160c), treated with ionomycin plus calcium $\left(\mathrm{I}+\mathrm{Ca}^{++}\right)$for the indicated time, and stained with the anti-FLAG antibody. Values are expressed as the percentage of cells with predominantly nuclear fluorescence. The proportion of cells with nuclear NF-ATc was also determined for cells treated with FK506 plus I $+\mathrm{Ca}^{++}$or with ionomycin plus $2.5 \mathrm{~mm}$ EGTA for 60 min. Efficient nuclear translocation of NF-ATc in COS cells requires both ionomycin and the elevation of extracellular calcium. The reason for this requirement for $\mathrm{Ca}^{2+}$ may be that ionomycin stimulation of COS cells does not result in an intracellular $\mathrm{Ca}^{2+}$ level as high as stimulated lymphocytes (R. Lewis, unpubl.). $(C)$ Analysis of subcellular localization of chimeric NF-AT(C $\Delta 418)$ GFP protein in nonstimulated (NS) cells or those treated with ionomycin plus calcium $\left(\mathrm{I}+\mathrm{Ca}^{++}\right)$. The position of the SRR and SP repeats is indicated. (D) Exit of NF-ATc ( $\square$ ) and NF-AT(C $\Delta 418$ )GFP $(O)$ from the nucleus. Transfected cells were stimulated with $\mathrm{I}+\mathrm{Ca}^{++}$for $1 \mathrm{hr}$ to localize the expressed protein in the nucleus. The medium was then replaced with that containing FK506. Slides were fixed at the indicated time, and the percentage of cells with cytoplasmic NF-ATc was determined. Cells expressing NF-ATc in the cytoplasm and those expressing NF-ATc in both cytoplasm and nucleus were added and divided by the total number of analyzed expressing cells. The error bars represent the standard deviation of the calculated value.

tion of NF-ATc (Fig. 3). Mutation of the sequence KRKK at position 682-685 to TRTG, or precise removal of these 4 residues, had no effect on nuclear localization of NFATc in response to $\mathrm{Ca}^{2+}$ elevation (data not shown). However, NF-ATc containing mutations in both regions remains cytoplasmic after ionomycin treatment. Thus, like other nuclear proteins with multiple NLSs (Richardson et al. 1986), the two NLSs are partially redundant, as suggested by the observation that either can direct cytoplasmic SOS to the nucleus but both must be mutated to prevent nuclear entry. These data also indicate that both NLSs must be inactive in the absence of $\mathrm{Ca}^{2+}$ stimulation.

Mutation of serines in the amino terminus leads to constitutive nuclear localization of NF-ATc

Because the amino terminus of NF-ATc is sufficient for calcineurin-dependent nuclear entry, we wished to de-

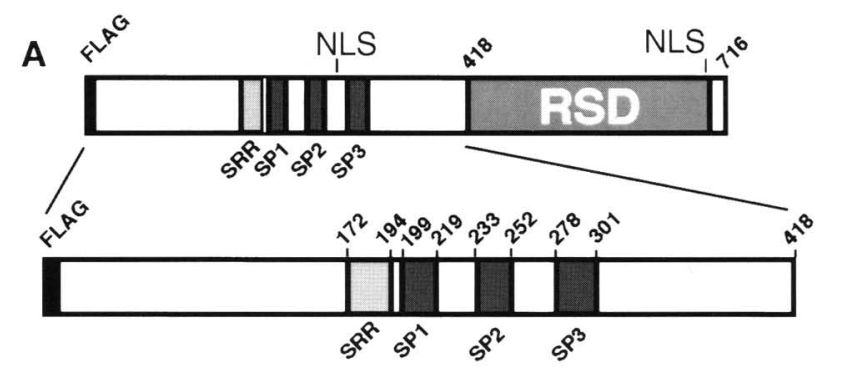

B

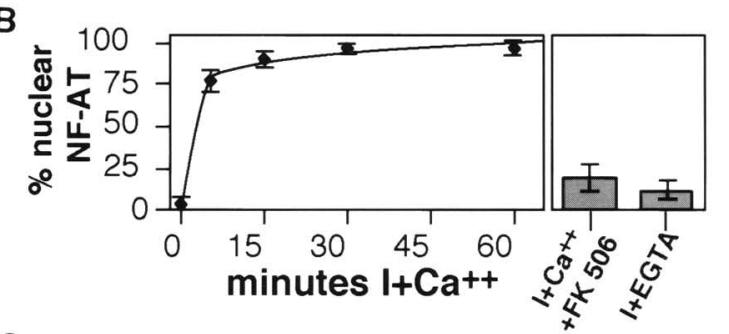

C
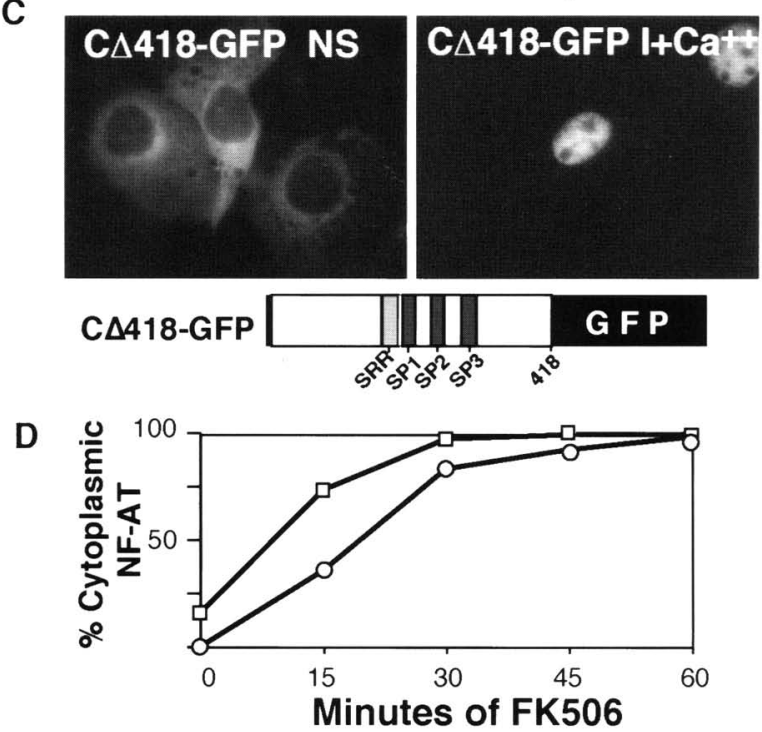

termine whether phosphorylation of the amino terminus directed subcellular compartmentalization of the transcription factor. The amino terminus of each NF-ATc protein contains three copies of a sequence that we call the SP-repeat motif (Ho et al. 1995; Hoey et al. 1995; Masuda et al. 1995). An additional SRR 23 amino acids in length lies just amino-terminal to the first SP repeat (Ho et al. 1995) (Fig. 1A). Phospho-amino acid analysis revealed that all phosphorylation is located on serines (N.A. Clipstone, unpubl.). Two-dimensional tryptic peptide maps show many phosphopeptides derived from the amino-terminal 418 amino acids (Beals et al. 1997). To determine whether phosphorylation of these particular serines could regulate NF-ATc localization we mutated select groups of conserved serines in the SRR and SP repeats to alanines and determined the subcellular localization of these mutants in COS cells. Mutation of all the serines within the SRR (mSRR) leads to constitutive nuclear localization in $100 \%$ of expressing cells that is 
A

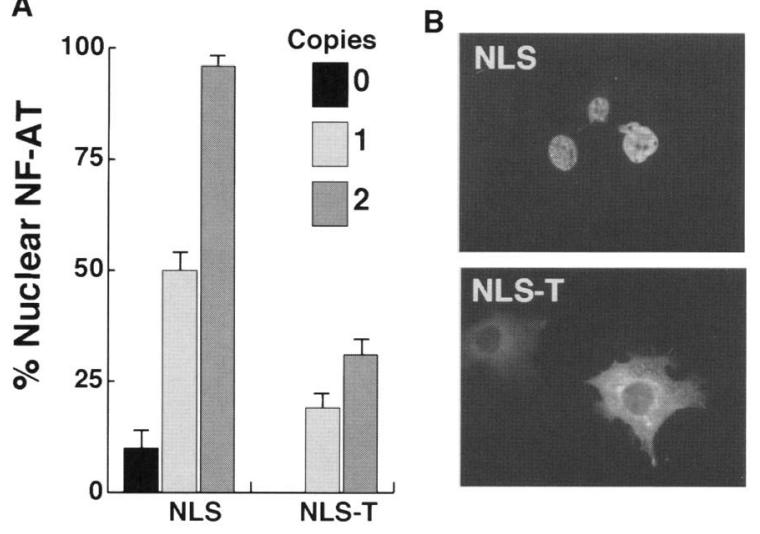

Figure 2. Addition of NLSs to NF-ATc results in constitutive nuclear localization. $(A)$ Analysis of nuclear localization of NFATc with wild-type or mutant NLS encoded at the amino terminus. COS cells were transiently transfected with NF-ATc expression constructs with zero, one, or two copies of either the NLS from SV40 large $T$ antigen (NLS) encoded between the FLAG epitope and the second amino acid of NF-ATc, or one or two copies of a mutant form of the NLS (NLS-T). Cells were stained with the anti-FLAG antibody. The percentage of unstimulated cells with nuclear fluorescence is indicated. $(B)$ Localization of NF-ATc in cells transfected with the expression construct containing two copies of the SV40 large T antigen (NLS) or with the expression construct containing two copies of NLS-T.

unaffected by FK506 (Fig. 4A). mSRR has an increased mobility on SDS electrophoresis, is present in the nucleus by Western blotting (Fig. 4B), and shows reduced incorporation of ${ }^{32} \mathrm{P}$ after in vivo labeling with orthophosphate (data not shown), consistent with the hypothesis that these serines affect the phosphorylation state of the protein in vivo. An NF-ATc mutant in which serines in the first SP repeat were substituted with alanines (mSPl) is also constitutively localized to the nucleus in $100 \%$ of expressing cells and shows a reduction in molecular weight (Fig. 4B). Similar results were obtained in NF-ATc mutants with $S \rightarrow$ A mutations in the first and third SP repeat $(\mathrm{mSP} 13)$ and in versions in which mutations were engineered in all three SP repeats (mSP123) or combined with the mutations in the SRR (mSRR + SP123) (data not shown). The subcellular localization of each of these mutant forms of NF-ATc is constitutively nuclear if they are expressed in Jurkat cells (data not shown), indicating that these phosphoserines control subcellular localization in a variety of cell types. The unregulated nuclear entry of the $S \rightarrow$ A mutations is not likely to be caused by denaturation of the protein, because each of these mutated forms of NF-ATc participate in NF-AT-dependent transcription in lymphocytes (C.R. Beals, unpubl.).

$S \rightarrow$ A mutation of the SRR results in the smallest alteration in apparent molecular weight, so we reasoned that this region might contain the smallest number of critical phosphoserines necessary for cytoplasmic localization. These mutants can be dephosphorylated further after transfection into cells and ionomycin treatment (Fig. 4B), indicating that the SRR mutant is still a substrate for a phosphatase, possibly calcineurin. We refined our analysis by mutation of smaller blocks of serines in the SRR. Alanine substitution at residues 172-176, 178181, and 184-188, but not residues 191-194, results in nuclear accumulation of NF-ATc in 100\% of expressing cells in the absence of $\mathrm{Ca}^{2+} /$ calcineurin signaling (Fig. $4 \mathrm{Cl}$. Interestingly, the mutants with constitutive nuclear localization remain in the nucleus after adding FK506, a treatment that leads to rapid cytoplasmic accumulation of wild-type NF-ATc, NF-ATp, or NF-ATC3 that has

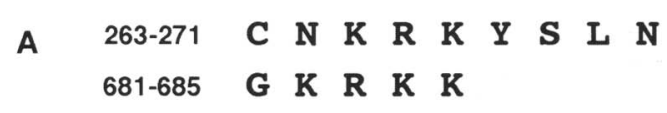

B
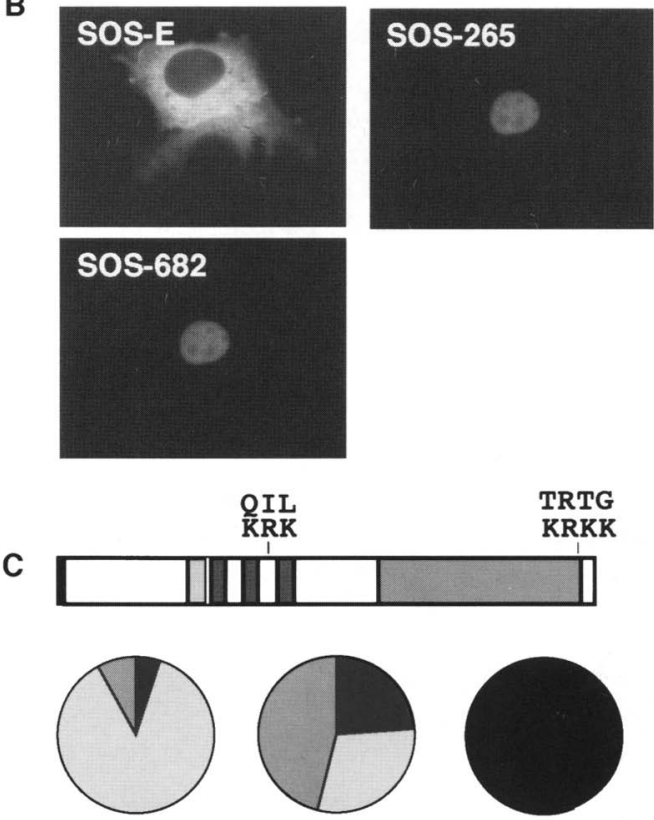

Wild Type

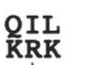

TRTG KRKK

Figure 3. Two partially redundant NLSs are necessary and sufficient for nuclear localization of NF-ATc. (A) Two conserved putative NLSs in NF-ATc are identified, and their positions in NF-ATc are indicated. $(B)$ Short sequences in NF-ATc redirect the cytoplasmic protein SOS to the nucleus. An expression construct encoding SOS (SOS-E) was expressed and visualized with $12 \mathrm{CA} 5$ antibody. Constructs encoding SOS-E attached to residues 263-271 of NF-ATc (SOS-265) or attached to residues 681685 of NF-ATc (SOS-682) were also detected with the 12CA5 antibody. (C) Nuclear localization of NF-ATc with one or more mutations in the NLSs. A diagram of the mutations made in the NLS in NF-ATc is above the wild-type sequence. The mutant expression constructs were transfected in COS cells, and the cells were stimulated with $\mathrm{I}+\mathrm{Ca}^{++}$for $60 \mathrm{~min}$. The percentage of cells staining in nucleus (lighted shaded areas), cytoplasm (solid areas), or both compartments (darkly shaded areas) was determined. $\mathrm{m} 265$ bears the mutation at residues $265-267$; $\mathrm{m} 265+682$ contains the $\mathrm{m} 265$ mutation as well as the mutation in residues $682-685$. 

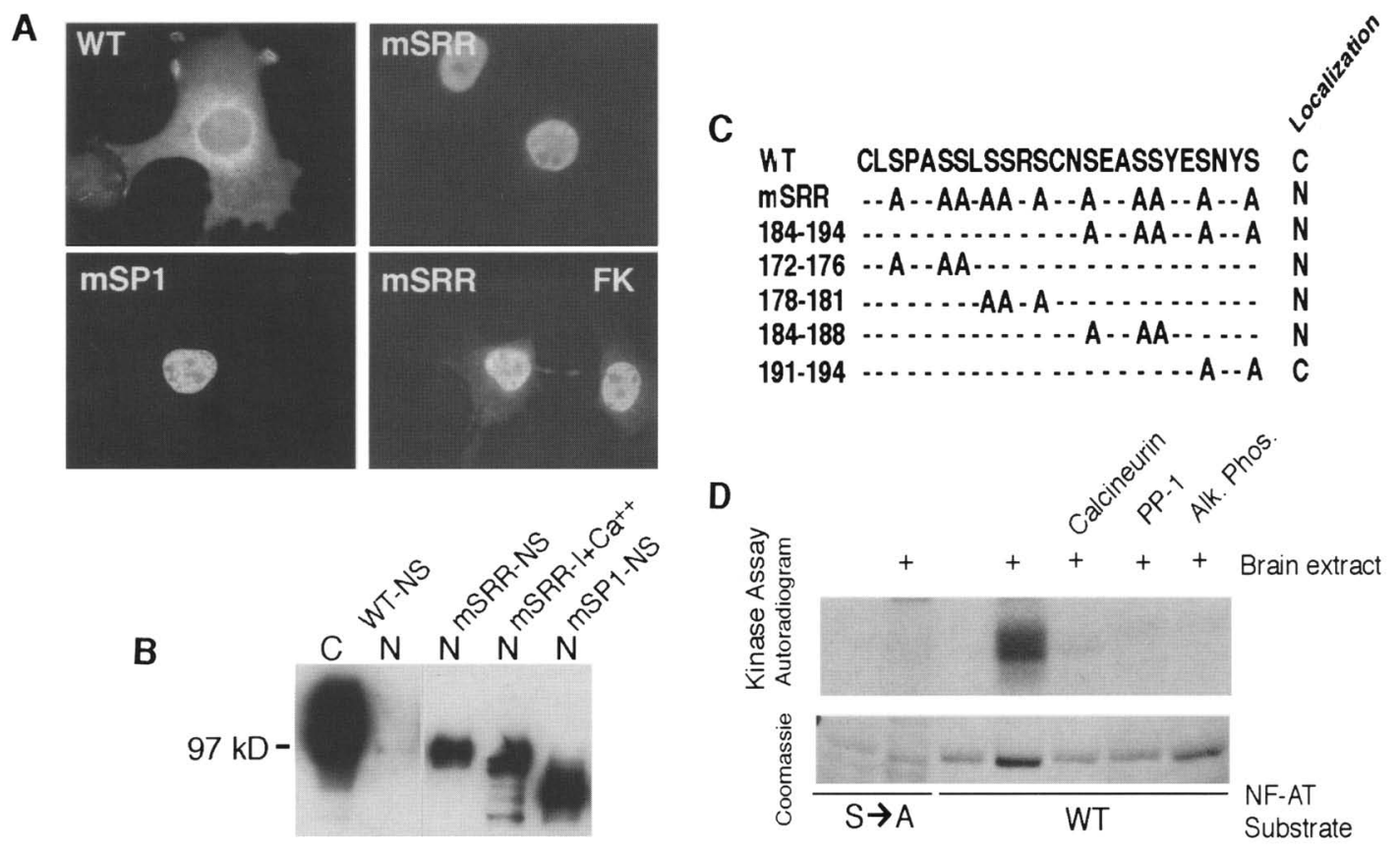

Figure 4. Serines in the SRR or first SP repeat results in constitutive nuclear localization and are substrates for calcineurin. $(A)$ Analysis of subcellular localization of wild-type and S $\rightarrow$ A mutations of NF-ATc. COS cells were transfected with wild-type (WT) NF-ATc expression constructs or with $\mathrm{S} \rightarrow \mathrm{A}$ mutations in the SRR (mSRR) or first SP repeat (mSP1) and left unstimulated or treated with FK506 (FK). (B) Immunoblot of cytoplasmic (C) or nuclear (N) extracts of cells transfected with wild-type (WT) or mutant forms of the NF-ATc cDNA. Transfected cells were treated for $60 \mathrm{~min}$ with media without additions (NS) or with ionomycin and calcium $\left(\mathrm{I}+\mathrm{Ca}^{++}\right)$and then separated into cytoplasmic and nuclear fractions and detected after electrophoresis with the anti-FLAG antibody. (C) Summary of the analysis of the nuclear localization of NF-ATc with mutations in the SRR. The sequence for each mutant is presented in single-letter code; (-) an identical residue. (Right) The localization of each mutant in transfected and unstimulated cells is indicated as cytoplasmic $(\mathrm{C})$ or nuclear $(\mathrm{N})$ localization. $(D)$ Conserved serines in the SP repeats are phosphorylated by cellular kinases and dephosphorylated by calcineurin. GST fusion proteins with amino acids 196-304 of NF-ATc (196-304 WT) or with S $\rightarrow$ A mutations in the three SP repeats $(196-304 \mathrm{~S} \rightarrow \mathrm{A})$ were phosphorylated by incubation with $\left[\gamma^{32} \mathrm{P}\right] \mathrm{ATP}$ and a partially purified preparation of cellular NF-AT kinase activity. After phosphorylation, the fusion proteins were washed to remove cellular proteins and incubated with phosphatases as indicated prior to separation by electrophoresis and autoradiography. Coomassie staining confirms that the substrate proteins are present.

been transported to the nucleus by stimulation (Flanagan et al. 1991; Shibasaki et al. 1996; Timmerman et al. 1996) (Fig. 4A). This result indicates that phosphorylation of these residues is necessary for export of NF-ATc from the nucleus.

To determine whether calcineurin could be the phosphatase directing nuclear entry, we made use of mutations in NF-AT at specific phosphoserines giving constitutive nuclear localization. We then determined whether calcineurin could specifically dephosphorylate the residues associated with nuclear entry. Amino acids 196304 of NF-ATc were expressed in bacteria as a glutathione S-transferase (GST) fusion protein [196-304 WT (wild-type)]. We also expressed a GST fusion protein in which conserved serines shown to be involved in nuclear import in the three SP repeats were changed to alanines (196-304 S $\rightarrow$ A). The difference in phosphorylation between these two proteins represents the specific phosphorylation of one or more of the conserved serines in the SP repeats. A brain extract containing the NF-AT kinase was used to phosphorylate NF-AT at approxi- mately seven phosphates per NF-AT on the same serines as used in vivo (Beals et al. 1997). The NF-ATc kinase activity was more active on the 196-304 WT substrate than the 196-304 S $\rightarrow$ A substrate (Fig. 4D). Once phosphorylated, the 196-304 WT substrate is readily dephosphorylated by in vitro treatment with several phosphatases, including calcineurin and phosphatase $I$, which is activated by calcineurin (Cohen 1989; Fig. 4D). These results indicate that the conserved serines in the SP repeats that control nuclear localization of NF-ATc are substrates for cellular kinases and calcineurin. Glycogen synthase kinase- 3 (GSK-3) is a highly conserved prolinedirected serine-threonine kinase that phosphorylates NF-AT in vivo and opposes $\mathrm{Ca}^{2+} /$ calcineurin-induced nuclear entry (Beals et al. 1997). GSK-3 phosphorylates the conserved serines in the SP repeats in vitro. The serines in the SRR and SP repeat motifs conform to a GSK-3 consensus substrate sequence (Fiol et al. 1994). Taken together, these results suggest that the conserved serines in these two motifs are phosphorylated in vivo by cellular kinases and dephosphorylated by calcineurin. 
Phosphoserines in the SRR control an intramolecular interaction within $N F-A T C$

The results described above raise the possibility that both basic NLSs interact with phosphoserines on the SP repeats and the serine-rich region to prevent nuclear entry in the unstimulated state. Such intramolecular interactions are difficult to discern because of the difficulty of expressing separate parts of the same protein at concentrations that would be equivalent to the high effective concentration of residues on the same peptide chain. To overcome this barrier to detecting intramolecular interactions, we immobilized one part of NF-ATc and looked for interactions with other regions expressed in extracts of COS cells, which phosphorylate and translocate NF-
ATc under $\mathrm{Ca}^{2+} /$ calcineurin control. When the carboxyl terminus of the protein containing the Rel similarity domain and one of the two partially redundant NLSs was immobilized (GST 415-716), it interacted readily and specifically with the amino-terminal half of NF-ATc (1418) when expressed in COS cell extracts (Fig. 5A), as well as interacting with the endogenous protein in extracts from lymphocytes (data not shown). Because the amino terminus, which contains multiple phosphoserines, might simply interact with basic residues in the Rel similarity region, we mutated the NLS in the rel similarity domain and examined binding of this mutated protein to amino-terminal residues $1-418$ (Fig. 5B). Mutation of the carboxy-terminal NLS from KRKK to TRTG abolished binding to the amino-terminal 418 residues.
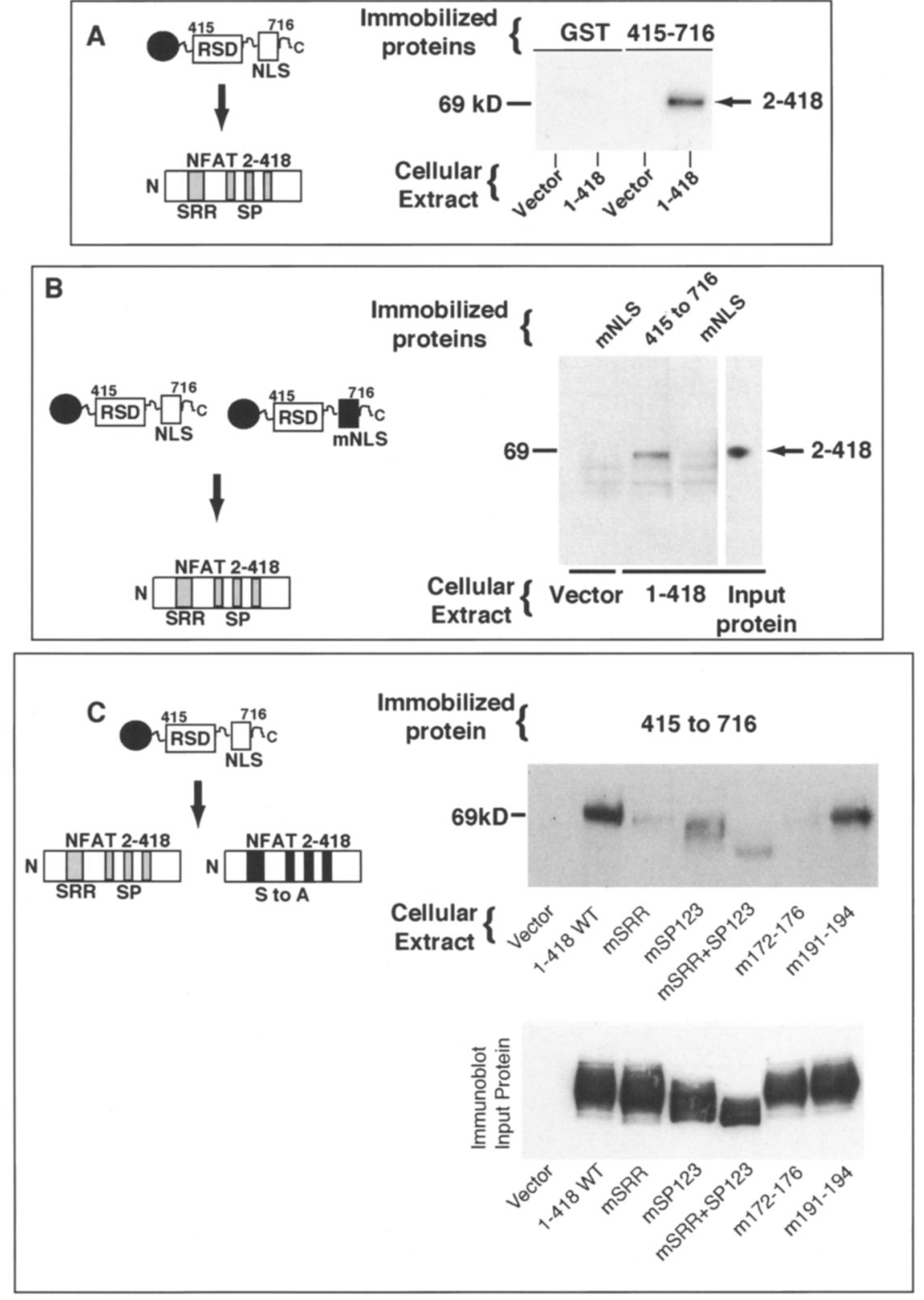

Figure 5. Interaction between the RSD of NF-ATc and the amino terminus of NFATc is dependent on the carboxy-terminal NLS and the SRR. (A) Extracts of COS cells that had been transfected with the empty expression vector (Vector) or a vector encoding the HA epitope-tagged amino-terminal 418 residues of NF-ATc (2-418) (Northrop et al. 1994) were incubated with glutathione-agarose beads coupled to GST or incubated with beads coupled to a GST fusion with the RSD of NF-ATc (GSTRSD). Affinity-selected proteins were detected by immunoblotting with the antiHA 12 CA5 antibody. $(B)$ COS cell extract containing 1-418 WT were incubated with GST-RSD or a version with a mutation in the carboxy-terminal NLS (mNLS) and bound proteins detected with the 12CA5 antibody. $(C)$ Extracts of COS cells that had been transfected with the HA epitopetagged amino-terminal 418 residues of NFATc (1-418 WT) or versions in which $\mathrm{S} \rightarrow \mathrm{A}$ mutations were present in the SRR or SP repeats were incubated with GSTRSD and then washed. The associated proteins were detected with the 7A6 antibody. The immunoblot shows the expression of the transfected expression constructs in 4 $\mu \mathrm{g}$ of cellular extract. 
This mutation is unlikely to result in denaturation because alteration of this NLS still permits cooperation with NF-ATn and NF-AT-dependent transcription in vivo (data not shown). Moreover, this interaction is sensitive to the presence of phosphoserines in the amino terminus (Fig. 5C). The amino-terminal 418 residues with $S \rightarrow$ A mutation in the SRR shows reduced association with the carboxyl terminus of the protein, whereas $\mathrm{S} \rightarrow \mathrm{A}$ changes in the three SP repeats affect this association less strongly. Each set of mutations results in more rapid migration on SDS electrophoresis, indicating that these $\mathrm{S} \rightarrow \mathrm{A}$ mutations prevent phosphorylation. Nonoverlapping $\mathrm{S} \rightarrow$ A mutations within the SRR (Fig. $4 \mathrm{C})$ were also tested in this intramolecular association assay. Alanine substitutions in residues 172-176 reduce the association with the rel similarity domain (RSD), whereas alanine substitutions in serines between 191 and 194 do not alter the association with the RSD. Interestingly, there is a correlation between binding to the RSD in the intramolecular association assay and $\mathrm{Ca}^{2+} /$ calcineurin-independent nuclear entry-m172-176 is constitutively nuclear and m191-194 undergoes regulated nuclear entry. The differences in the in vitro intramolecular association assay between each $\mathrm{S} \rightarrow \mathrm{A}$ mutations of NF-ATc are unlikely to be attributable to denaturation, as all mutants are immunoprecipitated by a monoclonal antibody to the region of NF-ATc in the SP repeats (Northrop et al. 1994), are stable when expressed in cells, and direct NF-AT-dependent transcription /data not shown). The binding activity is unlikely to indicate a head-to-tail dimer forming between full-length NFATc molecules, as the protein is a monomer in solution and when bound to DNA (Hoey et al. 1995). Thus, the interaction within NF-ATc is dependent on residues in the SRR as well as an intact carboxy-terminal NLS. The correlation between the subcellular localization of $\mathrm{mu}$ tants in the SRR and their behavior in in vitro binding assays (Figs. 4 and 5) suggests that this intramolecular association controls exposure and function of the carboxy-terminal NLS. The S $\rightarrow$ A changes in the three SP repeats disturbs binding to the RSD only weakly and suggests that these phosphoserines may not participate as strongly in the interaction with the carboxyl terminus of the protein. The dephosphorylation of the SP repeat motifs may result in nuclear localization by another mechanism, perhaps by exposure of the other NLS that lies between the second and third SP repeats.

\section{Discussion}

\section{Model of NF-ATc nuclear import}

Our studies support a model for NF-ATc translocation in which the function of two nuclear localization sequences is masked by phosphoserines in the SP repeats and the SRR, preventing nuclear localization of the phosphorylated protein. We propose that dephosphorylation alters an intramolecular association and exposes the NLSs leading to nuclear localization (Fig. 6). Precisely how the amino-terminal phosphoserines of NF-ATc

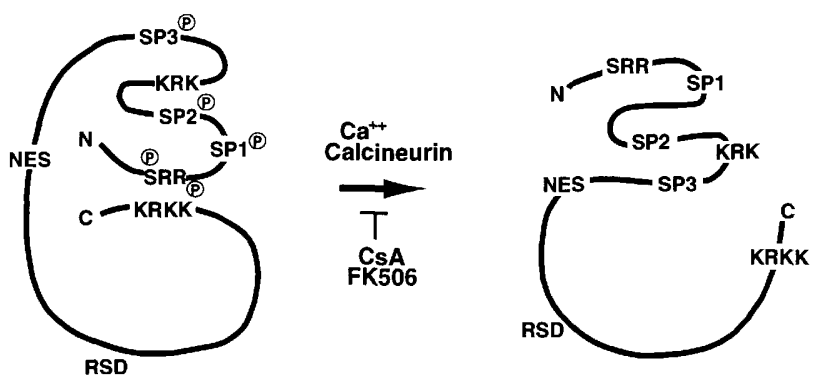

Figure 6. Model of the mechanism of NF-ATc nuclear entry. Cytoplasmic NF-ATc is phosphorylated on the SP repeats and SRR masking the activity of its two partially redundant NLSs, the sequence KRK at position 265-267, and the sequence KRKK at position 682-685. Dephosphorylation in response to activation of calcineurin leads to an alteration in an intramolecular interaction and perhaps a conformational change, exposing one or more of the NLSs to the nuclear import machinery. Once in the nucleus, termination of calcium signaling results in rapid export to the cytosol, possibly by the exposure of nuclear export sequences (NES).

mask the function of the NLSs is not yet certain, but an electrostatic interaction between the basic residues of the NLS and the phosphoserines is most likely, as we have demonstrated an intramolecular interaction dependent on the carboxy-terminal NLS and portions of the SRR. The model depicted in Figure 6 is likely to be extended to the other members of the NF-ATc gene family based on the conservation of the NLSs, the SRR, and the SP repeat regions. The amino terminus of NF-ATc3 and NF-ATp (Luo et al. 1996; Shibasaki et al. 1996) undergo $\mathrm{Ca}^{2+}$-sensitive nuclear entry.

NF-AT family members contain a region similar to the Rel family of transcriptional activators (Wolfe et al. 1997) suggesting that NF-AT might be retained in the cytoplasm through association with an IкB-like molecule. The NLS of NF-kB p65 is passively masked by its association with IкB (Baeuerle and Baltimore 1996) or with IкB-like sequences in the cytoplasmically localized precursor protein p105 (Rice et al. 1992). For NF-кB, nuclear entry ensues following proteolytic processing of either $I_{\kappa} B$ or of the ankyrin-repeat-containing regions of the p105 precursor. However, several observations indicate that the mechanism of NF-AT nuclear localization is different than NF- $\mathrm{kB}$. First, DNA-binding activity is not detectable after treating the cytoplasmic form of NFAT with nonionic detergents (Durand et al. 1988), as is the case for NF-kB. Second, none of the known IкB molecules are able to inhibit the function of NF-ATc in cotransfection assays (J. Northrop and G.R. Crabtree, unpubl.). Third, overexpressed NF-ATc is cytoplasmic, unlike the nuclear localization observed upon overexpression of the RelA/NF-кB p65 family members (Baeuerle and Baltimore 1996). Fourth, despite extensive efforts, we have been unable to detect an interaction of cytoplasmic NF-AT with another molecule with characteristics similar to an IкB (C.R. Beals and G.R. Crabtree, unpubl.). Finally, the sequences within NF- $\mathrm{B}$ family 
members that interact with IкB are not conserved in NFAT family members.

All NF-AT family members isolated so far contain two unique motifs, the SRR and the SP repeats. Our studies indicate that these sequences regulate the function or exposure of two partially redundant NLS elements in NF-ATc. NF-ATc proteins bearing $\mathrm{S} \rightarrow \mathrm{A}$ mutations in these motifs are phosphorylated less intensely and are localized to the nucleus in the absence of $\mathrm{Ca}^{2+}$ stimulation, suggesting that dephosphorylation of these residues leads to nuclear entry. NF-ATc, NF-ATp, and NF-ATc3 all return rapidly to the cytoplasm once $\mathrm{Ca}^{2+}$ levels are reduced or signaling is interrupted. Surprisingly, S $\rightarrow$ A mutations in these motifs prevent nuclear export of NFATc, as they remain nuclear in the presence of FK506, a treatment that causes the wild-type protein to return rapidly to the cytoplasm. Thus, our data indicate that the SRR and SP repeats are involved in nuclear import after activation and export from the nucleus once signaling is terminated. Glycogen synthase kinase-3 phosphorylates conserved serines in the SP repeats and promotes nuclear export of NF-AT, opposing $\mathrm{Ca}^{2+} /$ calcineurin signaling (Beals et al. 1997).

The nature of the dephosphorylation-dependent change leading to nuclear entry could be either dissolution of salt bridges or a conformation change, or both. The observation that several different $\mathrm{S} \rightarrow$ A mutations render NF-AT constitutively nuclear suggests that NFATc must assume a highly organized structure to completely mask the activities of two redundant NLSs. Many independent dephosphorylation events could alter this structure, exposing one or more of the redundant NLSs leading to $\mathrm{Ca}^{2+}$-independent, cyclosporin-resistant nuclear entry. The observation by Rao and colleagues that the cytoplasmic, phosphorylated form of NF-ATp binds DNA less well is also consistent with a conformational change in these proteins (Loh et al. 1996). In vitro binding experiments indicate that an intramolecular association between phosphoserines in the SRR motif may control exposure of the carboxy-terminal NLS. The phosphoserines in the SP repeats appear not to participate in this interaction, and the nuclear localization that results from $S \rightarrow A$ mutations in this motif may result from exposure of the other NLSs that lies between the second and third SP repeat.

Considerable evidence suggests that nuclear compartmentalization of NF-AT is the major mechanism by which NF-AT family members are regulated. First, all NF-AT family members isolated thus far are initially cytoplasmic and translocate to the nucleus after stimuli that increase cytoplasmic $\mathrm{Ca}^{2+}$ levels (Flanagan et al. 1991; Shaw et al. 1995; Timmerman et al. 1996). Second, the phosphorylated cytoplasmic form of NF-ATc specifically binds DNA and is capable of directing in vitro transcription when complemented with a nuclear extract of PMA-stimulated cells as a source of the nuclear component of NF-AT, NF-ATn (Flanagan et al. 1991). Third, deliberate nuclear localization of NF-AT bypasses the need for a $\mathrm{Ca}^{2+}$ stimulus for NF-AT-dependent transcription and IL-2 production (Timmerman et al. 1996). Fi- nally, cells in which NF-AT is expressed constitutively in the nucleus are resistant to the actions of cyclosporin A and FK506, at least in the sense that the endogenous IL-2 gene is activated in the presence of these drugs (Timmerman et al. 1996). Consequently, the details of the mechanism of NF-AT nuclear localization defines new targets for the development of immunosuppressive drugs.

NF-AT proteins appear to be expressed in the cytoplasm of most cell types including excitable cells such as neurons and muscle cells, as well as mast cells that release intracellular granules in response to transient $\mathrm{Ca}^{2+}$ elevation. Recent studies indicates that although NFATc translocates to the nucleus rapidly in response to brief calcium elevation, it will remain in the nucleus only if the level of $\mathrm{Ca}^{2+} /$ calcineurin signaling is maintained (Timmerman et al. 1996). The rapid shuttling of NF-ATc may underlie the way that cells discriminate transient changes in intracellular $\mathrm{Ca}^{2+}$ concentration that give rise to events such as exocytosis, adhesion, and synaptic transmission from the more sustained increases that participate in cell proliferation and differentiation. The intramolecular mechanism of control of nuclear localization that we have described is well suited to allow rapid transit of NF-ATc between the cytoplasm and the nucleus. This follows from the fact that intramolecular interactions are generally much faster than interactions between different molecules. Nuclear localization via an intramolecular interaction allows rapid changes in gene expression in response to the wide variety of stimuli that regulate intracellular $\mathrm{Ca}^{2+}$ in cell types as diverse as lymphocytes and neurons.

\section{Materials and methods}

\section{Materials}

Ionomycin was obtained from Calbiochem and dissolved in DMSO. FK506 was a gift from Fukisawa (Chicago, IL) and dissolved in ethanol. Cell culture media were from Sigma.

\section{DNA expression constructs}

Expression constructs used for in vivo subcellular localization of NF-ATc are based on pSH160c encoding the FLAG epitope tag (Ho et al. 1995) inserted at an $\mathrm{XbaI}$ site immediately $5^{\prime}$ to the second codon of the human NF-ATc1 cDNA in the pBI5 vector (Northrop et al. 1994). The constructs bearing the SV40 NLS and mutant (NLS-T) were created by insertion of synthetic oligonucleotides at the $X b a \mathrm{I}$ site of pSH160c. The inserted NLS is CTAGTCCTAAGAAGAAGAGAAAGGTAT; the sequence of NLS-T is CTAGTCCTAAGACGAAGAGAAAGGTAT and substitutes a threonine for a lysine (Kalderon et al. 1984). All point substitutions were created by sequential overlap extension PCR (Ho et al. 1989). The SRR was mutagenized by changing the 11 serines to alanines in residues 172-194 to form mSRR. Further mutations in the SRR were created as described in Figure $4 \mathrm{C}$. The first SP repeat was mutagenized by changing four serines to alanines in residues 199-211, mSPl. The second SP repeat was mutagenized by changing serines at 233 and 237 to alanines, $\mathrm{mSP} 2$. The third SP repeat was mutagenized at five serines at 278,282, 286, 290, and 299, mSP3. Carboxy-terminal deletion constructs to amino acid 418 were made with a hem- 
agglutinin (HA) epitope tag at the amino terminus and are based on pSH102c $\Delta 418$ (Northrop et al. 1994). The $\mathrm{S} \rightarrow \mathrm{A}$ substitutions made in the SRR and SP repeats were subcloned into this deletion construct. The NLS at residues $265-268$ was changed to QIL. The NLS at residues $682-685$ was changed to TRTG. The SOS expression constructs were based on the human SOS cDNA tagged at the carboxyl terminus by a HA epitope, pSOS-E (Holsinger et al. 1995). pSOS-265 was created by insertion of an oligonucleotides encoding the sequence LECNKRKYSLNVD at the unique SaII site between SOS and the HA epitope. The pSOS- 682 construct was created by inserting the sequence encoding LEGKRKKVD. The pSH160c $\Delta 418-G F P$ construct was made by fusing the BamHI-NotI fragment encoding GFP from pEGFP-1 (Clonetech) following the PvuII site at codon 418 of SH160c. pSH172 (GST-RSD), a fusion protein between GST and residues 415-716 of NF-ATc, was derived from DT102, which was constructed by inserting into the BamHI-EcoRI sites of pGEX-3X (Pharmacia) a PCR-amplified fragment of NFATc1 in which a BamHI site was placed $5^{\prime}$ to codon 415 in the appropriate frame and an EcoRI site placed $3^{\prime}$ to codon 591 . To create the full-length RSD (amino acids 415-716) as a GST fusion, the NotI-EcoRI fragment of pSH160c was then inserted into similarly digested pDT102, creating pSH172. A mutant form of SH172 in which the NLS at residues $682-685$ was changed to TRTG, creating mNLS. All PCR-generated constructs were verified by DNA sequencing.

\section{Cells culture and transfection}

COS-7 cells were maintained in Dulbecco's modified Eagle medium (DMEM) with $10 \%$ fetal calf serum (FCS), $100 \mu \mathrm{g} / \mathrm{ml}$ of penicillin $\mathrm{G}, 100 \mu \mathrm{g} / \mathrm{ml}$ of streptomycin, and $10 \mathrm{mM}$ HEPES $/ \mathrm{pH}$ 7.4) at $37^{\circ} \mathrm{C}$ in $5 \% \mathrm{CO}_{2}$. Cells were transfected by electroporation with $1 \mu \mathrm{g}$ of plasmid DNA (Clipstone and Crabtree 1992), plated on glass coverslips, and stimulated 18-24 hr post-transfection in fresh media or fresh media supplemented with drugs as described in the figure legends. Ionomycin was used at a final concentration of $2 \mu \mathrm{M}, \mathrm{CaCl}_{2}$ was added to $10 \mathrm{~mm}$, FK506 was used at $2 \mathrm{ng} / \mathrm{ml}$. FK506 was added at $2 \mathrm{ng} / \mathrm{ml} 15 \mathrm{~min}$ prior to addition of calcium and ionomycin. Cells were incubated at $37^{\circ} \mathrm{C}$ for $60 \mathrm{~min}$ unless indicated otherwise.

\section{Immunofluorescence}

Cells adhering to coverslips were fixed in $4 \%$ paraformaldehyde and permeabilized in $0.1 \%$ Triton X-100. The FLAG epitope was detected by incubating with $1 \mu \mathrm{g} / \mathrm{ml}$ of anti-FLAG M2 antibody (Eastman Kodak Co.), whereas the HA epitope was detected by incubating with 1:2000 dilution of 12CA5 ascites. These monoclonal antibodies were detected by incubation with biotin-conjugated anti-mouse IgG (Caltag), followed by streptavidin-FITC and DAPI (Molecular Probes). Fluorescence was visualization with a Zeiss Axiophot fluorescence microscope. The NFAT(C 4418$)$-GFP chimera was detected using its autofluorescence. Fluorescent cells in which the nucleus and plasma membrane could be identified were scored as containing predominantly cytoplasmic staining, predominantly nuclear staining, or both cytoplasmic and nuclear staining. At least 100 cells were scored on each coverslip. Cells undergoing mitosis or with multiple nuclei were excluded. For all deletion constructs, the subcellular localization was confirmed using a confocal imaging fluorescence microscope.

\section{Immunoblotting}

Cytoplasmic and nuclear extracts of COS cells were prepared (Ho et al. 1995) and subjected to SDS-PAGE. Western blotting was performed using the M2 or 12CA5 antibody and detected with anti-mouse peroxidase and chemiluminescence (Amersham).

\section{Protein interaction}

A GST fusion protein consisting of the Rel domain of NFATc1, GST-RSD (residues 415-716), was expressed in bacteria and affinity purified on glutathione-agarose (Smith and Johnson 1988). Residues $1-418$ of NF-ATc1 tagged at the amino terminus with the HA epitope (Northrop et al. 1994) were expressed in COS cells and an extract made by lysis in buffer A (Ho et al. 1995) with protease and phosphatase inhibitors. One hundred micrograms of this extract was incubated with $30 \mu \mathrm{l}$ of glutathione-agarose coupled to GST, GST-RSD, or GST-mNLS $1 \sim 2$ $\mu \mathrm{g}$ of fusion protein) in $300 \mu \mathrm{l}$ of incubation buffer $(50 \mathrm{~mm}$ HEPES at $\mathrm{pH} 7.8,150 \mathrm{~mm} \mathrm{NaCl}, 1 \mathrm{~mm}$ EDTA, $50 \mathrm{~mm} \mathrm{NaPO}$, $0.5 \%$ NP-40/ with protease and phosphatase inhibitors as in $\mathrm{Ho}$ et al. (1995) for $2 \mathrm{hr}$ at $4^{\circ} \mathrm{C}$ and washed three times in incubation buffer. Affinity-selected proteins were eluted from the washed beads with SDS sample buffer and detected by immunoblotting using either the 7A6 (Northrop et al. 1994) or 12CA5 monoclonal antibodies.

\section{In vitro dephosphorylation}

Residues 196-304 of NF-ATc1 (Northrop et al. 1994) were cloned into the SmaI site of pGEX-3X to generate pGSP. A GST fusion protein in which the $\mathrm{S} \rightarrow$ A substitutions in all three SP repeats described above was similarly constructed, pGAP, with $9 \mathrm{~S}$ and $10 \mathrm{~T}$ residues remaining. For kinase assays, $1 \mu \mathrm{gg}$ of fusion protein immobilized on glutathione-Sepharose with whole brain extract ( $55 \mu \mathrm{g}$ protein) (C.R. Beals and G. Crabtree, unpubl. / was incubated with $100 \mu \mathrm{M}$ ATP and $\left[\gamma^{32} \mathrm{P}\right] \mathrm{ATP} / 400$ $\mu \mathrm{Ci} / \mu$ mole $)$ in $50 \mu \mathrm{l}$ of kinase buffer $(20 \mathrm{~mm}$ Tris at $\mathrm{pH} 7.5,10$ $\mathrm{mM} \mathrm{MgCl}_{2}, 1 \mathrm{mM} \mathrm{DTT}$ ) for $30 \mathrm{~min}$ at $30^{\circ} \mathrm{C}$. Kinase reactions were terminated by washing the agarose beads three times in 1 $\mathrm{ml}$ of calcineurin buffer and then dephosphorylated with calcineurin as above, or treated with 2 units of shrimp alkaline phosphatase (U.S. Biochemical) or 5 units of protein phosphatase I (Boehringer Mannheim) in the buffer described by the manufacturer for $30 \mathrm{~min}$ at $30^{\circ} \mathrm{C}$. Samples were then electrophoresed and exposed for autoradiography.

\section{Acknowledgments}

We thank Steve Biggar, Roger Briesewitz, Leslie Holsinger, Luika Timmerman, and Hank Bayle for their comments on these experiments. C.R.B. was supported by a Howard Hughes Postdoctoral Fellowship for Physicians.

The publication costs of this article were defrayed in part by payment of page charges. This article must therefore be hereby marked "advertisement" in accordance with 18 USC section 1734 solely to indicate this fact.

\section{References}

Baeuerle, P.A. and D. Baltimore. 1996. NF-кB: Ten years after. Cell 87: 13-20.

Beals, C.R., C.M. Sheridan, C.W. Turck, P. Gardner, and G.R. Crabtree. 1997. Nuclear export of NF-ATc enhanced by glycogen synthase kinase-3. Science (in press).

Brunner, T., R.J. Mogil, D. LaFace, N.J. Yoo, A. Mahboubi, F. Echeverri, S.J. Martin, W.R. Force, D.H. Lynch, and C.F. Ware. 1995. Cell-autonomous Fas (CD95)/Fas-ligand inter- 
action mediates activation-induced apoptosis in T-cell hybridomas. Nature 373: 441-444.

Chang, H.Y., K. Takei, A.M. Sydor, T. Born, F. Rusnak, and D.G. Jay. 1995. Asymmetric retraction of growth cone filopodia following focal inactivation of calcineurin. Nature 376: 686690.

Chuvpilo, S., C. Schomberg, R. Gerwig, A. Heinfling, R. Reeves, F. Grummt, and E. Serfling. 1993. Multiple closely-linked NFAT/octamer and HMG I/Y) binding sites are part of the interleukin-4 promoter. Nucleic Acids Res. 21: 5694-5704.

Clipstone, N.A. and G.R. Crabtree. 1992. Identification of calcineurin as a key signalling enzyme in $\mathrm{T}$ cell activation. Nature 357: 695-697.

Cockerill, P.N., A.G. Bert, F. Jenkins, G.R. Ryan, M.F. Shannon, and M.A. Vadas. 1995. Human granulocyte-macrophage colony-stimulating factor enhancer function is associated with cooperative interactions between AP-1 and NFATp/c. Mol. Cell. Biol. 15: 2071-2079.

Cohen, P. 1989. The structure and regulation of protein phosphatases. Annu. Rev. Biochem. 58: 453-508.

Downward, J., J.D. Graves, P.H. Warne, S. Rayter, and D.A. Cantrell. 1990. Stimulation of p21ras upon T-cell activation. Nature 346: 719-723.

Durand, D.B., J.P. Shaw, M.R. Bush, R.E. Replogle, R. Belageje, and G.R. Crabtree. 1988. Characterization of antigen receptor response elements within the interleukin 2 enhancer. Mol. Cell. Biol. 8: 1715-1724.

Emmel, E.A., C.L. Verweij, D.B. Durand, K.M. Higgins, E. Lacy, and G.R. Crabtree. 1989. Cyclosporin A specifically inhibits function of nuclear proteins involved in $\mathrm{T}$ cell activation. Science 246: 1617-1620.

Fiol, C.J., J.S. Williams, C.H. Chou, Q.M. Wang, P.J. Roach, and O.M. Andrisani. 1994. A secondary phosphorylation of CREB341 at Ser129 is required for the cAMP-mediated control of gene expression. A role for glycogen synthase kinase-3 in the control of gene expression. J. Biol. Chem. 269: $32187-$ 32197.

Flanagan, W.M., B. Corthesy, R.J. Bram, and G.R. Crabtree. 1991. Nuclear association of a T-cell transcription factor blocked by FK-506 and cyclosporin A. Nature 352: 803-807.

Fruman, D.A., S.Y. Pai, S.J. Burakoff, and B.E. Bierer. 1995. Characterization of a mutant calcineurin A alpha gene expressed by EL4 lymphoma cells. Mol. Cell. Biol. 15: 38573863.

Goldfeld, A.E., P.G. McCaffrey, J.L. Strominger, and A. Rao. 1993. Identification of a novel cyclosporin-sensitive element in the human tumor necrosis factor $\alpha$ gene promoter. I. Exp. Med. 178: 1365-1379.

Ho, S.N., H.D. Hunt, R.M. Horton, J.K. Pullen, and L.R. Pease. 1989. Site-directed mutagensis by overlap extension using the polymerase chain reaction. Gene 77: 51-59.

Ho, S.N., D.J. Thomas, L.A. Timmerman, X. Li, U. Francke, and G.R. Crabtree. 1995. NFATc3, a lymphoid-specific NFATc family member that is calcium-regulated and exhibits distinct DNA binding specificity. J. Biol. Chem. 270: 1989819900.

Hodge, M.R., A.M. Ranger, F. Charles de la Brousse, T. Hoey, M.J. Grusby, and L.H. Glimcher. 1996. Hyperproliferation and dysregulation of IL-4 expression in NF-ATp-deficient mice. Immunity 4: 397-405.

Hoey, T., Y.-L. Sun, K. Williamson, and X. Xu. 1995. Isolation of two new members of thr NF-AT gene family and functional characterization of the NF-AT proteins. Immunity 2: 461472.

Holsinger, L.J., D.M. Spencer, D.J. Austin, S.L. Schreiber, and G.R. Crabtree. 1995. Signal transduction in T lymphocytes using a conditional allele of Sos. Proc. Natl. Acad. Sci. 92: 9810-9814.

Jain, J., P.G. McCaffrey, Z. Miner, T.K. Kerppola, J.N. Lambert, G.L. Verdine, T. Curran, and A. Rao. 1993. The T-cell transcription factor NFATp is a substrate for calcineurin and interacts with Fos and Jun. Nature 365: 352-355.

Kalderon, D., B.L. Roberts, W.D. Richardson, and A.E. Smith. 1984. A short amino acid sequence able to specify nuclear location. Cell 39: 499-509.

Klee, C.B., T.H. Crouch, and M.H. Krinks. 1979. Calcineurin: A calcium- and calmodulin-binding protein of the nervous system. Proc. Nat1. Acad. Sci. 76: 6270-6273.

Liu, J., J.D. Farmer, Jr., W.S. Lane, J. Friedman, I. Weissman, and S.L. Schreiber. 1991. Calcineurin is a common target of cyclophilin-cyclosporin A and FKBP-FK506 complexes. Cell 66: 807-815.

Loh, C., K.T. Shaw, J. Carew, J.P. Viola, C. Luo, B.A. Perrino, and A. Rao. 1996. Calcineurin binds the transcription factor NFAT1 and reversibly regulates its activity. J. Biol. Chem. 271: 10884-10891.

Luan, S., W. Li, F. Rusnak, S.M. Assmann, and S.L. Schreiber. 1993. Immunosuppressants implicate protein phosphatase regulation of $\mathrm{K}^{+}$channels in guard cells. Proc. Natl. Acad. Sci. 90: 2202-2206.

Luo, C., K.T.-Y. Shaw, A. Raghavan, J. Aramburu, F. GarciaCozar, B.A. Perrino, P.G. Hogan, and A. Rao. 1996. Interaction of calcineurin with a domain of the transcription factor NFAT1 that controls nuclear import. Proc. Natl. Acad. Sci. 93: 8907-8912.

Masuda, E.S., Y. Naito, H. Tokumitsu, D. Campbell, F. Saito, C. Hannum, K. Arai, and N. Arai. 1995. NFATx, a novel member of the nuclear factor of activated $T$ cells family that is expressed predominantly in the thymus. Mol. Cell. Biol. 15: $2697-2706$.

Minden, A., A. Lin, F.X. Claret, A. Abo, and M. Karin. 1995. Selective activation of the JNK signaling cascade and c-Jun transcriptional activity by the small GTPases Rac and Cdc42Hs. Cell 81: 1147-1157.

Northrop, J.P., S.N. Ho, L. Chen, D.J. Thomas, L.A. Timmerman, G.P. Nolan, A. Admon, and G.R. Crabtree. 1994. NFAT components define a family of transcription factors targeted in T-cell activation. Nature 369: 497-502.

O'Keefe, S.J., J. Tamura, R.L. Kincaid, M.J. Tocci, and E.A. O'Neill. 1992. FK506 and CsA-sensitive activation of the interleukin-2 promoter by calcineurin. Nature 357: 692-695.

Rice, N.R., M.L. MacKichan, and A. Israel. 1992. The precursor of NF-к B p50 has I kappa B-like functions. Cell 71: 243-253.

Richardson, W.D., B.L. Roberts, and A.E. Smith. 1986. Nuclear location signals in polyoma virus large-T. Cell 44: 77-85.

Roberts, B.L., W.D. Richardson, and A.E. Smith. 1987. The effect of protein context on nuclear location signal function. Cell 50: 465-475.

Rooney, J.W., T. Hoey, and L.H. Glimcher. 1995. Coordinate and cooperative roles for NF-AT and AP-1 in the regulation of the murine IL-4 gene. Immunity 2: 473-483.

Ruff, V.A. and K.L. Leach. 1995. Direct demonstration of NFATp dephosphorylation and nuclear localization in activated HT-2 cells using a specific NFATp polyclonal antibody. I. Biol. Chem. 270: 22602-22607.

Shaw, J.-P., P.J. Utz, D.B. Durand, J.J. Toole, E.A. Emmel, and G.R. Crabtree. 1988. Identification of a putative regulator of early $\mathrm{T}$ cell activation genes. Science 241: 202-205.

Shaw, K.T.-Y., A.M. Ho, A. Raghavan, J. Kim, J. Jain, J. Park, S. Sharma, A. Rao, and P.G. Hogan. 1995. Immunosuppressive drugs prevent a rapid dephosphorylation of transcription factor NFAT1 in stimulated immune cells. Proc. Natl. Acad. 
Beals et al.

Sci. 92: 11205-11209.

Shibasaki, F., E.R. Price, D. Milan, and F. McKeon. 1996. Role of kinases and the phosphatase calcineurin in the nuclear shuttling of transcription factor NF-AT4. Nature 382: 370-373.

Smith, D.B. and K.S. Johnson. 1988. Single-step purification of polypeptides expressed in Escherichia coli as fusions with glutathione-S-transferase. Gene 67: 31-40.

Stowers, L., D. Yelon, L.J. Berg, and J. Chant. 1995. Regulation of the polarization of $\mathrm{T}$ cells toward antigen-presenting cells by Ras-related GTPase CDC42. Proc. Natl. Acad. Sci. 92: 5027-5031.

Timmerman, L.A., N.A. Clipstone, S.N. Ho, J.P. Northrop, and G.R. Crabtree. 1996. Rapid shuttling of NF-AT in discrimination of $\mathrm{Ca}^{2+}$ signals and immunosuppression. Nature 383: $837-840$.

Treisman, R. 1995. Journey to the surface of the cell: Fos regulation and the SRE. EMBO I. 14: 4905-4913.

Wolfe, S.A. P. Zhou, V. Dotsch, L. Chen, A. You, S.A. Ho, G.R. Crabtree, G. Wagner, and G.L. Verdine. 1997. Unusual Rellike architecture in the DNA binding domain of the transcription factor NF-ATc. Nature 385: 172-176.

Woodrow, M., N.A. Clipstone, and D. Cantrell. 1993. p21 ras and calcineurin synergize to regulate the nuclear factor of activated T cells. J. Exp. Med. 178: 1517-1522.

Zhang, B.W., G. Zimmer, J. Chen, D. Ladd, E. Li, F.W. Alt, G. Wiederrecht, J. Cryan, E.A. O'Neill, C.E. Seidman, A.K. Abbas, and J.G. Seidman. 1996. T cell responses in calcineurin A $\alpha$-deficient mice. J. Exp. Med. 183: 413-420. 


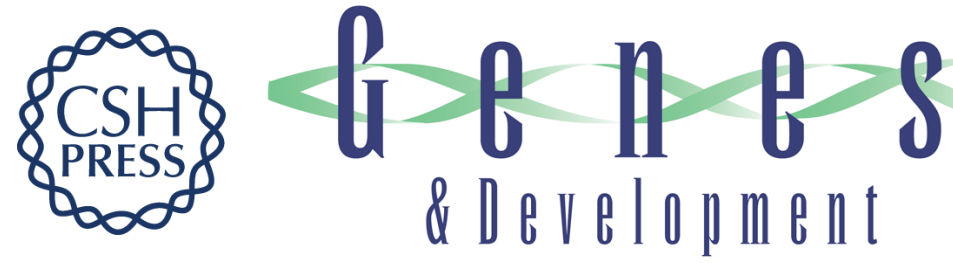

\section{Nuclear localization of NF-ATc by a calcineurin-dependent, cyclosporin-sensitive intramolecular interaction.}

C R Beals, N A Clipstone, S N Ho, et al.

Genes Dev. 1997, 11:

Access the most recent version at doi:10.1101/gad.11.7.824

References This article cites 45 articles, 19 of which can be accessed free at: http://genesdev.cshlp.org/content/11/7/824.full.html\#ref-list-1

License

Email Alerting

Service

Receive free email alerts when new articles cite this article - sign up in the box at the top right corner of the article or click here.

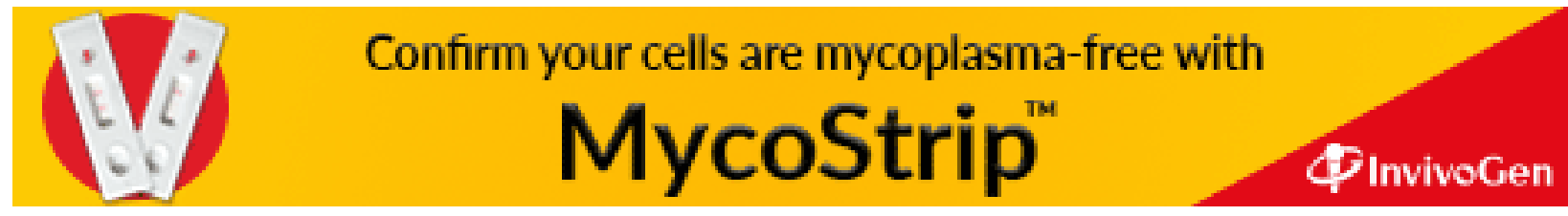

